\title{
Hospital Location Selection Using Spherical Fuzzy TOPSIS
}

\author{
Cengiz Kahraman ${ }^{\mathrm{a}}$, Fatma Kutlu Gündogdu ${ }^{\mathrm{a}, \mathrm{b}}$, Sezi Cevik Onarª Basar Oztaysi $^{\mathrm{a}}$ \\ a Industrial Engineering Department, Istanbul Technical University, Besiktas, Istanbul, 34367, Turkey \\ ${ }^{b}$ Industrial Engineering Department, Istanbul Kültür University, Bakırkoy, Istanbul, 34191, Turkey \\ kahramanc@itu.edu.tr, f.kutlu@iku.edu.tr, oztaysib@itu.edu.tr, sezi@outlook.com
}

\begin{abstract}
The three dimensional extensions of ordinary fuzzy sets such as intuitionistic fuzzy sets of type-2 (IFS2) or Pythagorean fuzzy sets (PFS), and neutrosophic sets (NS) aim at collecting experts' judgments more informatively and explicitly. In the literature, generalized three-dimensional spherical fuzzy sets have been introduced by Kutlu Gündoğdu and Kahraman [1]. In this paper, we develop and use spherical fuzzy TOPSIS and apply it to a hospital location selection problem.
\end{abstract}

Keywords: Hospital location selection, fuzzy MCDM, spherical fuzzy sets, TOPSIS

\section{Introduction}

TOPSIS method was introduced by Hwang and Yoon [2] in order to determine the best alternative among a set of alternatives in terms of a set of predefined criteria. Hwang and Yoon [2] introduced the concept of ideal solution, which is defined as the nearest to the positive ideal solution (PIS) to maximize the benefit type of attributes and the farthest from the negative ideal solution (NIS) to minimize the cost type of attributes. TOPSIS have been extensively applied in various decision making fields.

After the presentation of ordinary fuzzy sets by Zadeh [3], they have been very popular in almost all branches of science. Various researchers have developed several extensions of ordinary fuzzy sets. In recent years, several researchers have utilized these extensions in the solution of multi-criteria decision making problems. A classification of some recent representative publications with respect to the types of fuzzy extensions is as follows:

Type-2 fuzzy sets (T2FS): The concept of a type-2 fuzzy set was introduced by Zadeh [4] as an extension of the concept of an ordinary fuzzy set called a type-1 fuzzy set. Such sets are fuzzy sets whose membership grades themselves are type-1 fuzzy sets; they are very useful in circumstances where it is difficult to determine an exact membership function for a fuzzy set.

Intuitionistic fuzzy sets (IFS): Intuitionistic fuzzy sets introduced by Atanassov [5] enable defining both the membership and non-membership degrees of an element in a fuzzy set.

Hesitant fuzzy sets (HFS): Hesitant fuzzy sets can be used as a functional tool allowing many potential degrees of membership of an element to a set. These fuzzy sets force the membership degree of an element to be possible values between zero and one [6].

Pythagorean fuzzy sets (PFS): Atanassov's intuitionistic fuzzy sets of second type (IFS2) have been renamed by Yager [7] as Pythagorean fuzzy sets (PFS). Hence PFS and IFS2 mean the same fuzzy sets thereafter. Atanassov's intuitionistic fuzzy sets of second type (IFS2) or Yager's Pythagorean fuzzy sets are characterized by a membership degree and a nonmembership degree satisfying the condition that the square sum of its membership degree and nonmembership degree is equal to or less than one, which is a generalization of Intuitionistic Fuzzy Sets (IFS).

Neutrosophic sets (NS): Smarandache [8] developed neutrosophic logic and neutrosophic sets (NSs) as an extension of intuitionistic fuzzy sets. The neutrosophic set is defined as the set where each element of the universe has a degree of truthiness, indeterminacy and falsity.

Hospital location selection is a multicriteria decision making problem including several criteria that may be conflicting and dependent or independent. Hospital location selection is the application area of the developed SF-TOPSIS method in this paper.

The originality of the paper comes from its presentation of a novel SF-TOPSIS and the application of the proposed method to hospital location selection problem. The SF-TOPSIS enables decision makers to independently reflect their hesitancies in the decision process by using a linguistic evaluation scale based on spherical fuzzy sets.

The rest of this paper is organized as follows. Section 2 defines the hospital location selection 
problem. Section 3 summarizes the preliminaries spherical fuzzy sets. Spherical fuzzy TOPSIS is given in Section 4. Section 5 applies SF-TOPSIS method to a hospital location selection problem. Finally, the study is concluded in the last section.

\section{Hospital Location Selection Problem}

Hospitals should be safe and remain functional in emergencies and disasters. Proper selection of a hospital location has a direct effect on survival of affected population in disasters as well as cost and benefit of the hospital in non-emergency situation. To provide a chance for having equitable access to hospitals, a convenient location of establishing the service centers is of great importance.

Selecting a new hospital location is a serious decision for many urban planners and policy makers. In addition, this process is inherently complicated and time consuming. Furthermore, any fault in this decision may cause enormous cost. Decision makers have to evaluate and selecting the alternatives with respect to many quantitative and qualitative criteria under uncertainty. For these reasons, multi-criteria decision making (MCDM) under fuzzy environment is a good approach to improve precision of decision making and reduce waste of resources.

The criteria for a hospital location selection may be cost of land, land topography, building cost, population density, education level, economic conditions, proximity to transport, availability of infrastructure, and proximity to markets. With increasing demand on hospital beds, planning for expansion at a future date should always be kept in mind.

$\mathrm{Wu}$ et al. [9] present an analytic hierarchy process (AHP)-based evaluation model for hospital location selectin in Taiwan. The study adopts the modified Delphi method, the AHP and a sensitivity analysis to develop an evaluation method for selecting the optimal location of a regional hospital in Taiwan to determine its effectiveness. Vahidnia et al. [10] handle the specific problem of creating a welldistributed network of hospitals that delivers its services to the target population with minimal time, pollution and cost. They develop a Multi-Criteria Decision Analysis process that combines Geographical Information System (GIS) analysis with the Fuzzy Analytical Hierarchy Process (FAHP), and use this process to determine the optimum site for a new hospital in the Tehran urban area. Liu and Tsai [11] present location choices for foreign direct investments in new hospitals in China as a multi-criterion decision-making (MCDM) problem and develop a multidirectional relationship decision model combines the techniques of analytic network process
(ANP) and technique for order performance based on similarity to the ideal solution (TOPSIS).

\section{Spherical Fuzzy Sets}

Intuitionistic and Pythagorean fuzzy membership functions are composed of membership and nonmembership parameters. Hesitancy parameters can be calculated by $\pi_{i}^{n}=1-\mu-\vartheta$ for IFS and $\pi_{\tilde{p}}=\left(1-\mu_{\tilde{p}}^{2}(u)-v_{\tilde{p}}^{2}(u)\right)^{1 / 2}$ for IFS2 or PFS. Neutrosophic membership functions are also defined by three parameters truthiness, falsity and indeterminacy, whose sum can be between 0 and 3, and the value of each is between 0 and 1 independently. In spherical fuzzy sets, while the squared sum of membership, non-membership and hesitancy parameters can be between 0 and 1 , each of them can be defined between 0 and 1 independently to satisfy that their squared sum is at most equal to 1 . Figure 1 illustrates the differences among IFS, PFS, NS and SFS.

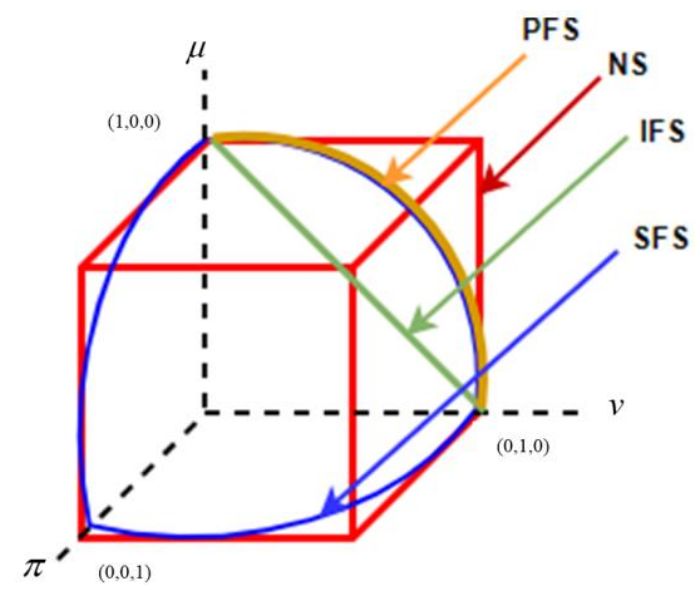

Fig. 1. Geometric representations of IFS, PFS, NS and SFS

A spherical fuzzy set $A_{S}$ of the universe of discourse $\mathrm{U}$ is given by

$$
\tilde{A}_{S}=\left\{\left\langle u,\left(\mu_{\tilde{A}_{S}}(u), v_{\tilde{A}_{S}}(u), \pi_{\tilde{A}_{S}}(u)\right)\right| u \in U\right\}
$$

where

$\mu_{\tilde{A}_{S}}: U \rightarrow[0,1], v_{\tilde{A}_{S}}(u): U \rightarrow[0,1], \pi_{\tilde{A}_{S}}: U \rightarrow[0,1]$

and

$0 \leq \mu_{\tilde{A}_{S}}^{2}(u)+v_{\tilde{A}_{S}}^{2}(u)+\pi_{\tilde{A}_{S}}^{2}(u) \leq 1 \quad \forall u \in U$

For each $u$, the numbers $\mu_{\tilde{A}_{S}}(u), v_{\tilde{A}_{S}}(u)$ and $\pi_{\tilde{A}_{S}}(u)$ are the degree of membership, nonmembership and hesitancy of $u$ to $A_{S}$, respectively [1]. 
On the basis of relationship between SFS and PFS, Kutlu Gündoğdu \& Kahraman [1] further define some novel operations for SFS as below:

Definition 1: Addition and multiplication of two spherical fuzzy numbers, multiplication by a scalar number, and power operation for spherical fuzzy numbers are presented below, respectively.

$$
\begin{aligned}
& \tilde{A}_{S} \oplus \tilde{B}_{S}=\left\{\begin{array}{l}
\left(\mu_{\tilde{A}_{S}}^{2}+\mu_{\tilde{B}_{S}}^{2}-\mu_{\tilde{A}_{S}}^{2} \mu_{\tilde{B}_{S}}^{2}\right)^{1 / 2}, v_{\tilde{A}_{S}} v_{\tilde{B}_{S}}, \\
\left(\left(1-\mu_{\tilde{B}_{S}}^{2}\right) \pi_{\tilde{A}_{S}}^{2}+\left(1-\mu_{\tilde{A}_{S}}^{2}\right) \pi_{\tilde{B}_{S}}^{2}-\pi_{\tilde{A}_{S}}^{2} \pi_{\tilde{B}_{S}}^{2}\right)^{1 / 2}
\end{array}\right\} \\
& \tilde{A}_{S} \otimes \tilde{B}_{S}=\left\{\begin{array}{l}
\mu_{\tilde{A}_{S}} \mu_{\tilde{B}_{S}},\left(v_{\tilde{A}_{S}}^{2}+v_{\tilde{B}_{S}}^{2}-v_{\tilde{A}_{S}}^{2} v_{\tilde{B}_{S}}^{2}\right)^{1 / 2}, \\
\left(\left(1-v_{\tilde{B}_{S}}^{2}\right) \pi_{\tilde{A}_{S}}^{2}+\left(1-v_{\tilde{A}_{S}}^{2}\right) \pi_{\tilde{B}_{S}}^{2}-\pi_{\tilde{A}_{S}}^{2} \pi_{\tilde{B}_{S}}^{2}\right)^{1 / 2}
\end{array}\right\}
\end{aligned}
$$

$\lambda \cdot \tilde{A}_{S}=\left\{\begin{array}{l}\left(1-\left(1-\mu_{\tilde{A}_{S}}^{2}\right)^{\lambda}\right)^{1 / 2}, v_{\tilde{A}_{S}}^{\lambda}, \\ \left(\left(1-\mu_{\tilde{A}_{S}}^{2}\right)^{\lambda}-\left(1-\mu_{\tilde{A}_{S}}^{2}-\pi_{\tilde{A}_{S}}^{2}\right)^{\lambda}\right)^{1 / 2}\end{array}\right\} \lambda>0$

$$
\tilde{A}_{S}^{\lambda}=\left\{\begin{array}{l}
\mu_{\tilde{A}_{S}}^{\lambda},\left(1-\left(1-v_{\tilde{A}_{S}}^{2}\right)^{\lambda}\right)^{1 / 2}, \\
\left(\left(1-v_{\tilde{A}_{S}}^{2}\right)^{\lambda}-\left(1-v_{\tilde{A}_{S}}^{2}-\pi_{\tilde{A}_{S}}^{2}\right)^{\lambda}\right)^{1 / 2}
\end{array}\right\} \lambda>0
$$

Definition 2: Spherical Weighted Arithmetic Mean (SWAM) with respect to, $w=\left(w_{1}, w_{2} \ldots . . ., w_{n}\right) ; w_{i} \in[0,1] ; \sum_{i=1}^{n} w_{i}=1$,

SWAM is defined as;

$$
\begin{aligned}
& \text { SWAM }{ }_{w}\left(\tilde{A}_{S 1}, \ldots \ldots . ., \tilde{A}_{S n}\right)=w_{1} \tilde{A}_{S 1}+w_{2} \tilde{A}_{S 2}+\ldots \ldots .+w_{n} \tilde{A}_{S n} \\
& =\left\{\begin{array}{l}
{\left[1-\prod_{i=1}^{n}\left(1-\mu_{\tilde{A}_{s}}^{2}\right)^{w_{i}}\right]^{1 / 2}, \prod_{i=1}^{n} v_{\tilde{A}_{S}}^{w_{i}},} \\
{\left[\prod_{i=1}^{n}\left(1-\mu_{\tilde{A}_{S}}^{2}\right)^{w_{i}}-\prod_{i=1}^{n}\left(1-\mu_{\tilde{A}_{S}}^{2}-\pi_{\tilde{A}_{S i}}^{2}\right)^{w_{i}}\right]^{1 / 2}}
\end{array}\right\}
\end{aligned}
$$

Spherical Weighted Geometric Mean (SWGM) with respect

to, $w=\left(w_{1}, w_{2} \ldots . . ., w_{n}\right) ; w_{i} \in[0,1] ; \sum_{i=1}^{n} w_{i}=1$,

SWGM is defined as;

$$
\begin{aligned}
& S W G M_{w}\left(\tilde{A}_{1}, \ldots \ldots . ., \tilde{A}_{n}\right)=\tilde{A}_{S 1}{ }^{w_{1}}+\tilde{A}_{S 2}{ }^{w_{2}}+\ldots . .+\tilde{A}_{S n}{ }^{w_{n}} \\
& =\left\{\begin{array}{l}
\prod_{i=1}^{n} \mu_{\tilde{A}_{S i}}^{w_{i}},\left[1-\prod_{i=1}^{n}\left(1-v_{\tilde{A}_{S i}}^{2}\right)^{w_{i}}\right]^{1 / 2}, \\
{\left[\prod_{i=1}^{n}\left(1-v_{\tilde{A}_{S i}}^{2}\right)^{w_{i}}-\prod_{i=1}^{n}\left(1-v_{\tilde{A}_{S i}}^{2}-\pi_{\tilde{A}_{S i}}^{2}\right)^{w_{i}}\right]^{1 / 2}}
\end{array}\right\}
\end{aligned}
$$

\section{Spherical Fuzzy TOPSIS}

A MCDM problem can be expressed as a decision matrix whose elements indicate the evaluation values of all alternatives with respect to each criterion under Spherical fuzzy environment. Let $X=\left\{x_{1}, x_{2}, \ldots \ldots x_{m}\right\} \quad(m \geq 2)$ be a discrete set of $\mathrm{m}$ feasible alternatives and $C=\left\{C_{1}, C_{2}, \ldots \ldots C_{n}\right\}$ be a finite set of criteria, and $w=\left\{w_{1}, w_{2}, \ldots . . w_{n}\right\}$ be the weight vector of all criteria which satisfies $0 \leq w_{j} \leq 1$ and $\sum_{j=1}^{n} w_{j}=1$.

Step 1: Let DMs fill in the decision and criteria evaluation matrices using the linguistic terms given in Table 1.

Table 1

Linguistic terms and their corresponding spherical fuzzy numbers

\begin{tabular}{|l|c|}
\hline Linguistic Terms & $(\mu, v, \pi)$ \\
\hline Absolutely more Importance (AI) & $(0.9,0.1,0.1)$ \\
\hline Very High Importance (VHI) & $(0.8,0.2,0.2)$ \\
\hline High Importance (HI) & $(0.7,0.3,0.3)$ \\
\hline Slightly More Importance (SMI) & $(0.6,0.4,0.4)$ \\
\hline Equally Importance (EI) & $(0.5,0.5,0.5)$ \\
\hline Slightly Low Importance (SLI) & $(0.4,0.6,0.4)$ \\
\hline Low Importance (LI) & $(0.3,0.7,0.3)$ \\
\hline Very Low Importance (VLI) & $(0.2,0.8,0.2)$ \\
\hline Absolutely Low Importance (ALI) & $(0.1,0.9,0.1)$ \\
\hline
\end{tabular}

Step 2: Aggregate the judgments of each decision maker (DM) using Spherical Weighted Arithmetic Mean (SWAM) or Spherical Weighted Geometric Mean (SWGM) operators that are given in Eqs. (7) and (8).

Step 2.1: Aggregate the criteria weights.

Step 2.2: Construct aggregated spherical fuzzy decision matrix based on the opinions of decision makers.

Denote the evaluation values of Alternative $X_{i}(i=1,2 \ldots . . m)$ with respect to criterion $C_{j}(j=1,2 \ldots . n) \quad$ by $\quad C_{j}\left(X_{i}\right)=\left(\mu_{i j}, v_{i j}, \pi_{i j}\right) \quad$ and $D=\left(C_{j}\left(X_{i}\right)\right)_{m \times n}$ is a spherical fuzzy decision matrix. For a MCDM problem with SFS, decision matrix $D=\left(C_{j}\left(X_{i}\right)\right)_{m \times n}$ should be constructed as in Eq. (9). 
$D=\left(C_{j}\left(X_{i}\right)\right)_{m \times n}=\left(\begin{array}{llll}\left(\mu_{11}, v_{11}, \pi_{11}\right) & \left(\mu_{12}, v_{12}, \pi_{12}\right) & \ldots & \left(\mu_{1 n}, v_{1 n}, \pi_{1 n}\right) \\ \left(\mu_{21}, v_{21}, \pi_{21}\right) & \left(\mu_{22}, v_{22}, \pi_{22}\right) & \ldots & \left(\mu_{2 n}, v_{2 n}, \pi_{2 n}\right) \\ \cdot & \cdot & & \cdot \\ \cdot & \cdot & & \cdot \\ \cdot\left(\mu_{m 1}, v_{m 1}, \pi_{m 1}\right) & \left(\mu_{m 2}, v_{m 2}, \pi_{m 2}\right) & \ldots & \left(\mu_{m n}, v_{m n}, \pi_{m n}\right)\end{array}\right)$

Step 3: Construct the aggregated weighted spherical fuzzy decision matrix. After the weights of the criteria and the rating of the alternatives have been determined, the aggregated weighted spherical fuzzy decision matrix is constructed by utilizing Eq. (4) and then the aggregated weighted spherical fuzzy decision matrix can be defined as follows:

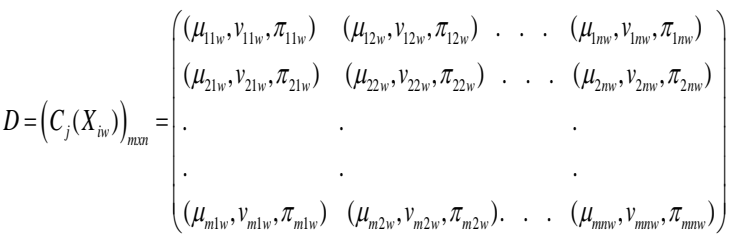

Step 4: Defuzzify the aggregated weighted decision matrix by using Eq. (11). This operation is needed only for determining the SF positive and negative ideal solutions. Later, we continue with the SF numbers in the next steps.

$$
\operatorname{Score}\left(C_{j}\left(X_{i w}\right)\right)=\left(2 \mu_{i j w}-\pi_{i j w} / 2\right)^{2}-\left(v_{i j w}-\pi_{i j w} / 2\right)^{2}
$$

Step 5: Determine the Spherical Fuzzy Positive Ideal Solution (SF-PIS) and the Spherical Fuzzy Negative Ideal Solution (SF-NIS), based on the score values obtained in Step 4.

For the SF-PIS:

$$
\begin{aligned}
& X^{*}=\left\{C_{j}, \max _{i}<\operatorname{Score}\left(C_{j}\left(X_{i w}\right)\right)>\mid j=1,2 \ldots n\right\} \\
& X^{*}=\left\{\left\langle C_{1},\left(\mu_{1}^{*}, v_{1}^{*}, \pi_{1}^{*}\right)\right\rangle,\left\langle C_{2},\left(\mu_{2}^{*}, v_{2}^{*}, \pi_{2}^{*}\right)\right\rangle \ldots .\left\langle C_{n},\left(\mu_{n}^{*}, v_{n}^{*}, \pi_{n}^{*}\right)\right\rangle\right\}
\end{aligned}
$$

For the SF-NIS:

$$
\begin{aligned}
& X^{-}=\left\{C_{j}, \min _{i}<\operatorname{Score}\left(C_{j}\left(X_{i w}\right)\right)>\mid j=1,2 \ldots n\right\} \\
& X^{-}=\left\{\left\langle C_{1},\left(\mu_{1}^{-}, v_{1}^{-}, \pi_{1}^{-}\right)\right\rangle,\left\langle C_{2},\left(\mu_{2}^{-}, v_{2}^{-}, \pi_{2}^{-}\right)\right\rangle \ldots .\left\langle C_{n},\left(\mu_{n}^{-}, v_{n}^{-}, \pi_{n}^{-}\right)\right\rangle\right\}
\end{aligned}
$$

Step 6: Calculate the distances between alternative $X_{i}$ and the SF-PIS as well as SF-NIS, respectively. Normalized Euclidean distance will be used for this step [11].

For the SF-NIS:

$$
D\left(X_{i}, X^{-}\right)=\sqrt{\frac{1}{2 n} \sum_{i=1}^{n}\left(\left(\mu_{X_{i}}-\mu_{X^{-}}\right)^{2}+\left(v_{X_{i}}-v_{X^{-}}\right)^{2}+\left(\pi_{X_{i}}-\pi_{X^{-}}\right)^{2}\right)}
$$

For the SF-PIS:

$$
D\left(X_{i}, X^{*}\right)=\sqrt{\frac{1}{2 n} \sum_{i=1}^{n}\left(\left(\mu_{X_{i}}-\mu_{X^{*}}\right)^{2}+\left(v_{X_{i}}-v_{X^{*}}\right)^{2}+\left(\pi_{X_{i}}-\pi_{X^{*}}\right)^{2}\right)}
$$

Step 7: Calculate the classical closeness ratio given in Eq. (16).

$$
\xi\left(X_{i}\right)=\frac{D\left(X_{i}, X^{-}\right)}{D\left(X_{i}, X^{*}\right)+D\left(X_{i}, X^{-}\right)}
$$

Step 8: Determine the optimal ranking order of the alternatives and identify the optimal alternative. We put the alternatives into orders with respect to the decreasing values of closeness ratio.

\section{An Application}

Our proposed methodology is implemented to a hospital location selection problem. Selection of hospital location decision criteria can vary depending on the number of qualitative and quantitative elements. For this goal, five locations $\left(\mathrm{X}_{1}, \mathrm{X}_{2}, \mathrm{X}_{3}\right.$, $\mathrm{X}_{4}$, and $\mathrm{X}_{5}$ ) are assessed. Based on a comprehensive literature review, a variety of criteria has been determined. In this illustrative example, five of these criteria are employed: Installation Costs $\left(\mathrm{C}_{1}\right)$, closeness to target area $\left(\mathrm{C}_{2}\right)$, environmental factors $\left(\mathrm{C}_{3}\right)$, demographic infrastructure $\left(\mathrm{C}_{4}\right)$ and transportation opportunities $\left(\mathrm{C}_{5}\right)$. In the evaluation process, three decision makers (DM1, DM2, and DM3) are included who are three experienced engineers in supply chain and logistics management. The weights of these decision makers who have different experience levels are $0.5,0.3$ and 0.2 , respectively. Firstly, the judgments are gathered from decision makers as linguistic terms given in Table 2 with respect to the goal. All judgments are given in Table 2. These judgments are aggregated using SWAM operator by considering the importance levels of decision makers. Decision matrices are obtained as in Table 3. The importance weights of the criteria assigned by DMs and represented by linguistic terms. After the weights of the criteria and the rating of the alternatives have been determined, the aggregated weighted spherical fuzzy decision matrices are constructed by utilizing Eq. (4) as given in Table 5. Based on Table 5 and Eq. (11), score function values are obtained as in Table 6. The highest values represent PIS while the lowest values represent NIS. According to the best and worst scores, the corresponding Spherical Fuzzy Positive Ideal Solution (SF-PIS) and Spherical Fuzzy Negative Ideal Solution (SF-NIS) are given in Table 7. In the next step, based on Eqs. (14) and (15), we can calculate the distances between alternative $X_{i}$ and the SF-PIS as well as SF-NIS, respectively. Based on Eq. (16), closeness ratios are calculated and presented in Table 8 . The closeness ratios indicate that the best alternative is $\mathrm{X}_{1}$ and overall ranking is $X_{1}>X_{3}>X_{5}>X_{4}>X_{2}$. 
Table 2. Judgments of DMs

\begin{tabular}{|c|c|c|c|c|c|c|c|c|c|c|c|c|c|c|c|c|c|}
\hline DM1 & $\mathrm{C}_{1}$ & $\mathrm{C}_{2}$ & $\mathrm{C}_{3}$ & $\mathrm{C}_{4}$ & $\mathrm{C}_{5}$ & DM2 & $\mathrm{C}_{1}$ & $\mathrm{C}_{2}$ & $\mathrm{C}_{3}$ & $\mathrm{C}_{4}$ & $\mathrm{C}_{5}$ & DM3 & $\mathrm{C}_{1}$ & $\mathrm{C}_{2}$ & $\mathrm{C}_{3}$ & $\mathrm{C}_{4}$ & $\mathrm{C}_{5}$ \\
\hline$X_{1}$ & $\mathrm{AI}$ & SMI & VHI & SLI & SMI & $\mathrm{X}_{1}$ & $\mathrm{HI}$ & SMI & VHI & EI & HI & $\mathrm{X}_{1}$ & EI & HI & $\mathrm{HI}$ & HI & SLI \\
\hline$X_{2}$ & SLI & VHI & HI & EI & VHI & $\mathrm{X}_{2}$ & SLI & HI & HI & HI & SLI & $\mathrm{X}_{2}$ & VHI & EI & EI & EI & EI \\
\hline$X_{3}$ & EI & VHI & VHI & HI & VHI & $X_{3}$ & SLI & VHI & $\mathrm{HI}$ & EI & SLI & $X_{3}$ & HI & VHI & VHI & HI & HI \\
\hline$X_{4}$ & $\mathrm{HI}$ & SMI & HI & EI & SMI & $X_{4}$ & SMI & HI & LI & LI & SMI & $\mathrm{X}_{4}$ & SMI & HI & LI & LI & EI \\
\hline $\mathrm{X}_{5}$ & $\mathrm{HI}$ & HI & LI & SMI & HI & $\mathrm{X}_{5}$ & $\mathrm{HI}$ & SMI & HI & SMI & HI & $\mathrm{X}_{5}$ & HI & EI & EI & EI & SMI \\
\hline
\end{tabular}

Table 3. Decision matrix by using SWAM operator

\begin{tabular}{cccccc}
\hline Alternatives & $\mathrm{C}_{1}$ & $\mathrm{C}_{2}$ & $\mathrm{C}_{3}$ & $\mathrm{C}_{4}$ & $\mathrm{C}_{5}$ \\
\hline $\mathrm{X}_{1}$ & $(0.81,0.19,0.23)$ & $(0.62,0.38,0.38)$ & $(0.78,0.22,0.22)$ & $(0.51,0.49,0.41)$ & $(0.61,0.40,0.37)$ \\
$\mathrm{X}_{2}$ & $(0.40,0.60,0.40)$ & $(0.73,0.27,0.29)$ & $(0.67,0.33,0.34)$ & $(0.58,0.43,0.44)$ \\
$\mathrm{X}_{3}$ & $(0.53,0.48,0.43)$ & $(0.80,0.20,0.20)$ & $(0.77,0.23,0.23)$ & $(0.65,0.35,0.36)$ & $(0.68,0.33,0.31)$ \\
$\mathrm{X}_{4}$ & $(0.65,0.35,0.35)$ & $(0.65,0.35,0.35)$ & $(0.56,0.46,0.31)$ & $(0.42,0.59,0.43)$ & $(0.58,0.42,0.42)$ \\
$\mathrm{X}_{5}$ & $(0.70,0.30,0.30)$ & $(0.64,0.36,0.37)$ & $(0.51,0.51,0.36)$ & $(0.58,0.42,0.42)$ & $(0.68,0.32,0.32)$ \\
\hline
\end{tabular}

Table 5. Weighted decision matrix based on SWAM operator

\begin{tabular}{cccccc}
\hline Alternatives & $\mathrm{C}_{1}$ & $\mathrm{C}_{2}$ & $\mathrm{C}_{3}$ & $\mathrm{C}_{4}$ & $\mathrm{C}_{5}$ \\
\hline $\mathrm{X}_{1}$ & $(0.66,0.27,0.31)$ & $(0.46,0.45,0.44)$ & $(0.61,0.31,0.31)$ & $(0.21,0.71,0.47)$ & $(0.41,0.49,0.44)$ \\
$\mathrm{X}_{2}$ & $(0.33,0.62,0.42)$ & $(0.54,0.38,0.38)$ & $(0.52,0.39,0.39)$ & $(0.24,0.69,0.49)$ & $(0.46,0.45,0.41)$ \\
$\mathrm{X}_{3}$ & $(0.43,0.51,0.46)$ & $(0.59,0.33,0.34)$ & $(0.60,0.32,0.31)$ & $(0.27,0.66,0.47)$ & $(0.48,0.43,0.39)$ \\
$\mathrm{X}_{4}$ & $(0.53,0.39,0.40)$ & $(0.48,0.43,0.42)$ & $(0.44,0.50,0.36)$ & $(0.17,0.76,0.46)$ & $(0.40,0.51,0.47)$ \\
$\mathrm{X}_{5}$ & $(0.57,0.35,0.36)$ & $(0.47,0.44,0.43)$ & $(0.40,0.54,0.39)$ & $(0.24,0.68,0.49)$ & $(0.47,0.44,0.42)$ \\
\hline
\end{tabular}

Table 7. SF-PIS and SF-NIS based on SWAM operator

\begin{tabular}{cccccc}
\hline & $\mathrm{C}_{1}$ & $\mathrm{C}_{2}$ & $\mathrm{C}_{3}$ & $\mathrm{C}_{4}$ & $\mathrm{C}_{5}$ \\
\hline $\mathrm{X}^{*}$ (Best) & $(0.66,0.27,0.31)$ & $(0.59,0.33,0,34)$ & $(0.61,0.31,0.31)$ & $(0.27,0.66,0.47)$ & $(0.48,0.43,0.39)$ \\
$\mathrm{X}^{-}$(Worst) & $(0.33,0.62,0.42)$ & $(0.46,0.45,0.44)$ & $(0.40,0.54,0.39)$ & $(0.17,0.76,0.46)$ & $(0.40,0.51,0.47)$ \\
\hline
\end{tabular}

Table 4. Weights of each criterion

\begin{tabular}{ccccc}
\hline Criteria & DM1 & DM2 & DM3 & Weights of each criterion \\
\hline $\mathrm{C}_{1}$ & AI & HI & EI & $(0.81,0.19,0.23)$ \\
$\mathrm{C}_{2}$ & VHI & HI & EI & $(0.73,0.27,0.29)$ \\
$\mathrm{C}_{3}$ & VHI & HI & VHI & $(0.77,0.23,0.23)$ \\
$\mathrm{C}_{4}$ & EI & LI & LI & $(0.42,0.59,0.43)$ \\
$\mathrm{C}_{5}$ & HI & HI & SMI & $(0.68,0.32,0.32)$ \\
\hline
\end{tabular}

Table 6. Score function values based on SWAM operator

\begin{tabular}{cccccc}
\hline Alternatives & $\mathrm{C}_{1}$ & $\mathrm{C}_{2}$ & $\mathrm{C}_{3}$ & $\mathrm{C}_{4}$ & $\mathrm{C}_{5}$ \\
\hline $\mathrm{X}_{1}$ & 1.361 & 0.427 & 1.103 & -0.191 & 0.296 \\
$\mathrm{X}_{2}$ & 0.028 & 0.745 & 0.673 & -0.138 & 0.463 \\
$\mathrm{X}_{3}$ & 0.327 & 0.981 & 1.067 & -0.080 & 0.543 \\
$\mathrm{X}_{4}$ & 0.718 & 0.514 & 0.382 & -0.269 & 0.238 \\
$\mathrm{X}_{5}$ & 0.894 & 0.471 & 0.240 & -0.133 & 0.472
\end{tabular}

Table 8. Distances to positive and negative ideal solutions

\begin{tabular}{ccccc}
\hline Alternatives & $D\left(X_{i}, X^{*}\right)$ & $D\left(X_{i}, X^{-}\right)$ & Closeness Ratio & Rank \\
\hline $\mathrm{X}_{1}$ & 0.078 & 0.190 & 0.710 & 1 \\
$\mathrm{X}_{2}$ & 0.168 & 0.087 & 0.341 & 5 \\
$\mathrm{X}_{3}$ & 0.115 & 0.145 & 0.557 & 2 \\
$\mathrm{X}_{4}$ & 0.133 & 0.101 & 0.432 & 4 \\
$\mathrm{X}_{5}$ & 0.127 & 0.127 & 0.499 & 3 \\
\hline
\end{tabular}

\section{Conclusion}

Spherical fuzzy sets are a new extension of ordinary fuzzy sets. They are based on the mixed idea of intuitionistic fuzzy sets of type 2 (Pythagorean fuzzy sets) and neutrosophic sets. Spherical fuzzy TOPSIS is also a new extension of classical TOPSIS method. 
The proposed SF TOPSIS method has been applied to a hospital location selection problem.

For further research, we suggest interval valued SF TOPSIS method to be developed and be compared with single valued SF TOPSIS method. Alternatively, different integrated fuzzy multicriteria decision making methodologies [12] or fuzzy mathematical programming techniques [13], or fuzzy statistical decision making approaches [14], [15] can be used for hospital location selection by spherical fuzzy sets. SF-WASPAS [16], SF-VIKOR [17], or Interval-valued SF-TOPSIS [18] methods can be used for the comparative analyses of hospital location selection problem.

\section{References}

[1] F. Kutlu Gündoğdu \& C. Kahraman, Spherical fuzzy sets and spherical fuzzy TOPSIS method. Journal of Intelligent \& Fuzzy Systems Preprint (2019) 36(1): 337-352.

[2] C. L. Hwang \& K. Yoon, Methods for multiple attribute decision making, In Multiple attribute decision making, Springer, Berlin, Heidelberg. 1981, pp. 58-191.

[3] L. A. Zadeh, Fuzzy Sets, Information and Control 8 (1965) 338-353.

[4] L. A. Zadeh, The concept of a linguistic variable and its application to approximate reasoning, Information sciences 8 (1975) 199-249.

[5] K. T. Atanassov, Intuitionistic fuzzy sets, Fuzzy sets and Systems 20(1) (1986) 87-96.

[6] V. Torra, Hesitant fuzzy sets. International Journal of Intelligent Systems 25(6) (2010) 529539.

[7] R. Yager, Pythagorean fuzzy subsets. Joint IFSA World Congress and NAFIPS Annual Meeting, Edmonton, Canada 2013, pp. 57-61.

[8] F. Smarandache, Neutrosophy: neutrosophic probability, set, and logic: analytic synthesis \& synthetic analysis (1998).

[9] C. R. Wu, C. T. Lin \& H. C. Chen, Optimal selection of location for Taiwanese hospitals to ensure a competitive advantage by using the analytic hierarchy process and sensitivity analysis. Building and environment 42(3) (2007) 1431-1444.

[10] M. H. Vahidnia, A. A. Alesheikh \& A. Alimohammadi, Hospital site selection using fuzzy AHP and its derivatives, Journal of environmental management 90(10) (2009) 30483056.

[11] C. T. Lin \& M. C. Tsai, Location choice for direct foreign investment in new hospitals in China by using ANP and TOPSIS. Quality \& Quantity 44(2) (2010) 375-390.

[12] Kaya, T., Kahraman, C.(2011) A fuzzy approach to e-banking website quality assessment based on an integrated AHP-ELECTRE method,
Technological and Economic Development of Economy, 17 (2), 313-334.

[13] Zhang, G., Lu, J. (2007) Model and approach of fuzzy bilevel decision making for logistics planning problem, Journal of Enterprise Information Management 20 (2), 178-197.

[14] Kaya, I., Kahraman, C. (2010) Development of fuzzy process accuracy index for decision making problems, Information Sciences 180 (6), 861-872

[15] Kaya,I., Kahraman, C. (2009) Fuzzy robust process capability indices for risk assessment of air pollution, Stochastic Environmental Research and Risk Assessment 23 (4), 529-541.

[16] F. Kutlu Gündoğdu \& C. Kahraman, Extension of WASPAS with spherical fuzzy sets. Informatica (2019) 30(2): 269-292.

[17] F. Kutlu Gündoğdu \& C. Kahraman, A novel VIKOR method using spherical fuzzy sets and its application to warehouse site selection. Journal of Intelligent \& Fuzzy Systems (2019) Pre-press.

[18] F. Kutlu Gündoğdu \& C. Kahraman, A novel fuzzy TOPSIS method using emerging intervalvalued spherical fuzzy sets. Engineering Applications and Artificial Intelligence (2019) Pre-press. 